\title{
LETTER \\ Block Adaptive Algorithm for Signal Declipping Based on Null Space Alternating Optimization
}

\author{
Tomohiro TAKAHASHI ${ }^{\dagger a}$, Student Member, Kazunori URUMA ${ }^{\dagger}$, Nonmember, \\ Katsumi KONISHI ${ }^{\dagger \dagger}$, and Toshihiro FURUKAWA ${ }^{\dagger}$, Members
}

\begin{abstract}
SUMMARY This letter deals with the signal declipping algorithm based on the matrix rank minimization approach, which can be applied to the signal restoration in linear systems. We focus on the null space of a low-rank matrix and provide a block adaptive algorithm of the matrix rank minimization approach to signal declipping based on the null space alternating optimization (NSAO) algorithm. Numerical examples show that the proposed algorithm is faster and has better performance than other algorithms.

key words: matrix rank minimization, signal declipping, signal restoration, block algorithm
\end{abstract}

\section{Introduction}

This letter proposes a block adaptive signal declipping algorithm based on the matrix rank minimization approach. Signal clipping is a signal distortion process that occurs when the signal goes beyond the clipping gain which is the input limit of a digital acquisition system. For example, telephony systems, low cost digital audio, photo and video recorder systems often suffer from distortion. Several algorithms have been proposed for the signal declipping [1]-[5]. The double sides period substitution method is proposed for packet voice communications [1]. The clipped samples are divided some blocks and substituted by neighborhood unclipped samples for recovering signal in this method. In the previous methods [2], [3], the signal declipping algorithms are proposed based on the assumption that the signal is modeled by the autoregressive (AR) model (and more statistical models). The time-varying AR coefficients and clipped samples are estimated by an EM-like alternating algorithm in [2]. The sparse representation based signal declipping algorithm is proposed in [4] using the orthogonal matching pursuit (OMP). The performance of the algorithm [4] is better than that of other conventional algorithms such as [2] when a desirable overcomplete dictionary is given. But the performance of the OMP based algorithm highly depends on given dictionaries. Finally, the matrix rank minimization approach to signal declipping is proposed based on the AR model [5]. The advantage of the rank minimization ap-

Manuscript received May 30, 2014.

Manuscript revised August 22, 2014.

Manuscript publicized October 6, 2014.

${ }^{\dagger}$ The authors are with the Department of Management Science, Tokyo University of Science, Tokyo, 162-8601 Japan.

${ }_{\dagger}$ The author is with the Department of Computer Science, Kogakuin University, Tokyo, 163-8677 Japan.

a)E-mail: takahashi@ms.kagu.tus.ac.jp

DOI: 10.1587/transinf.2014EDL8109 proach is that the signal is restored even if both the coefficients and an order of the AR model are unknown. The rank minimization approach is used in other signal restoration applications such as video inpainting [6] and image inpainting [7].

This letter also takes the rank minimization approach and modifies the algorithm proposed in [5] using block adaptive method to improve the quality of declipping and reduce the computing time. In speech and audio signal processing, we usually use the adaptive processing because the signal is not always stationary. The block adaptive algorithm is one of the most practical adaptive algorithms in signal processing and can be easily implemented in most algorithms. The method proposed in [5] does not consider adaptive processing. To improve convergence of the method, this letter uses the null space alternating optimization (NSAO) algorithm, which gives a low-rank solution by optimizing a null space matrix, and proposes a block adaptive algorithm reusing the optimized null space matrix in the previous block. Under the assumption that the difference of the AR coefficients between neighbor blocks is small, the difference between the null space matrices of neighbor block is also small, which motivates us to implement a block adaptive algorithm reusing the null space matrix. The contribution of this letter is to provide a block adaptive algorithm for rank minimization based signal declipping algorithm.

\section{Main Results}

\subsection{Problem Formulation}

Let $\left\{s_{i}\right\}_{i=1}^{L}$ and $\left\{y_{i}\right\}_{i=1}^{L}$ denote the undistorted signals and the observed signals with clipping, respectively, and satisfies the AR model, that is, $s_{i}$ is described as follows,

$$
s_{i}=\sum_{j=1}^{r} a_{j} s_{i-j},
$$

where $L, r$ and $a_{j}$ denote the signal length, the model order and $j$ th model coefficient, respectively. Without loss of generality, this letter normalizes $s_{i}$ such that $\max _{i}\left(\left|s_{i}\right|\right)=1$. The observed signal $y_{i}$ is described by the distortion function $g_{C}$ as

$$
y_{i}=g_{C}\left(s_{i}\right)= \begin{cases}C & \text { if } C<s_{i} \\ -C & \text { if } s_{i}<-C \\ s_{i} & \text { else }\end{cases}
$$


where $C$ is a constant corresponding to the clipping gain which is input limit of the digital system, which can be estimated easily from observed signal. In order to simplify the discussion, we consider here a background noise-free case.

To provide a block algorithm, we consider the signal declipping problem which recovers $\left\{s_{i}\right\}_{i=n}^{n+L_{b}}$ from $\left\{y_{i}\right\}_{i=n}^{n+L_{b}}$, where $L_{b}$ denotes the block length.

Then we focus on the fact that the model order of AR model is equal to the rank of Hankel matrix defined by

$$
S_{n}=\left[\begin{array}{cccc}
s_{n} & s_{n+1} & \ldots & s_{n+N-1} \\
s_{n+1} & s_{n+2} & \ldots & s_{n+N} \\
\vdots & \vdots & \ddots & \vdots \\
s_{n+N-1} & s_{n+N} & \ldots & s_{n+2 N-2}
\end{array}\right] \in \boldsymbol{R}^{N \times N}
$$

where $N=\left(L_{b}+1\right) / 2$ and propose a matrix rank minimization approach to signal declipping algorithm. Since it holds that rank $S_{n}=r$ if and only if the model order of the AR system (1) equals $r$, if model order $r$ is known, we can recover the signals by solving the following problem,

$$
\begin{array}{ll}
\text { find } & \hat{S}_{n} \in R^{N \times N} \\
\text { subject to } & \hat{S}_{n} \in \mathcal{H} \cap \mathcal{I}, \\
& \text { rank } \hat{S}_{n}=r,
\end{array}
$$

where $\hat{S}_{n}$ is a design variable, $\mathcal{H}$ denotes the set of matrices with the Hankel structure defined in (3), $I$ denotes the set of matrices defined by

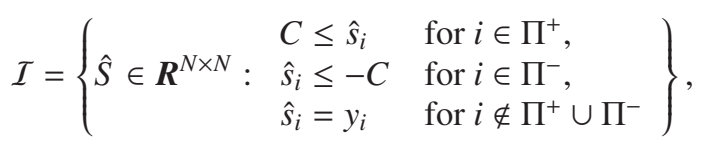

and $\Pi^{+}$and $\Pi^{-}$denote the index sets of positive and negative clipped samples in $y_{i}$, respectively. However the model order is unknown in general, and this letter formulates the signal declipping problem as the following matrix rank minimization problem to estimate the model order,

$$
\begin{array}{ll}
\text { Minimize } & \operatorname{rank} \hat{S}_{n} \\
\text { subject to } & \hat{S}_{n} \in \mathcal{H} \cap \mathcal{I} \in R^{N \times N} .
\end{array}
$$

Although the rank minimization problem is NP hard, several useful and practical algorithms are proposed to obtain its solution [8]-[10]. As mentioned in Sect.1, this letter uses the NSAO algorithm [9] since this algorithm is suitable for the block adaptive algorithm.

\subsection{Null Space Alternating Optimization Approach}

This subsection gives a simple description of the NSAO algorithm proposed in [9]. Since minimizing the rank of a matrix is equivalent to maximizing the rank of its null space matrix, (6) is equal to the following problem,

$$
\begin{array}{ll}
\underset{\hat{S}_{n}, W}{\operatorname{Maximize}} & \operatorname{rank} W \\
\text { subject to } & \hat{S}_{n} W=\mathbf{0}_{N, N}, \hat{S}_{n} \in \mathcal{H} \cap \mathcal{I}, W \in R^{N \times N},
\end{array}
$$

where $\mathbf{0}_{N, N}$ denotes the $N \times N$ zero matrix. If the sequence $s_{i}$ is completely modeled by the AR model, (7) has a solution such that $\hat{S}_{n} W=\mathbf{0}_{N, N}$. However, $s_{i}$ usually contains a model error, and therefore there seldom exists $W$ such that $\hat{S}_{n} W=\mathbf{0}_{N, N}$. To relax this difficulty, [9] proposes the NSAO algorithm, and we obtain the following relaxed problem by applying NSAO,

$$
\begin{array}{ll}
\underset{\hat{S}_{n}, W}{\operatorname{Minimize}} & \gamma\|W\|_{F}^{2}+\left\|\hat{S}_{n} W\right\|_{F}^{2} \\
\text { subject to } & \hat{S}_{n} \in \mathcal{H} \cap \mathcal{I}, W \in \mathcal{W}
\end{array},
$$

where $W$ is defined by

$$
\mathcal{W}=\left\{W \in \boldsymbol{R}^{N \times N}: W_{i, i}=1\right\},
$$

and $W_{i, i}$ denotes the $i$ th diagonal element of $W$. The key idea of this approach is relaxing the rank maximization problem by the Frobenius norm minimization. If there is no constraint except for $W_{i, i}=1$, we can exactly maximize the rank of $\mathrm{W}$ by minimizing its Frobenius norm, and the aim of (8) is to maximize the rank of $W$ and to force $\hat{S}_{n} W$ to zero matrix by minimizing their Frobenius norms. Because (8) is a convex problem and can be solved efficiently if we fix $\hat{S}_{n}$ or $W$ as a constant matrix, the NSAO algorithm solves (8) iteratively by fixing them as a constant matrix alternately. The NSAO algorithm solves (9) by fixing $W$ and $\hat{S}_{n}$ as a constant alternately and iteratively. Letting $Z=\hat{S}_{n}$ and $\Omega=\mathcal{H} \cap \mathcal{I}$ in the NSAO algorithm, a rank minimization based signal declipping algorithm is obtained as shown in Algorithm 1, where $P_{\mathcal{I}}$ and $P_{\mathcal{H}}$ denote the orthogonal projections onto $\mathcal{I}$ and $\mathcal{H}$, respectively.

\subsection{Block Adaptive Algorithm}

Next we consider the block adaptive algorithm. Since (8) is a nonconvex optimization, the quality of the solution and the convergence speed of Algorithm 1 depends on the initial value of $W$. To improve the algorithm, this letter proposes a block adaptive algorithm by giving better initial value in each block.

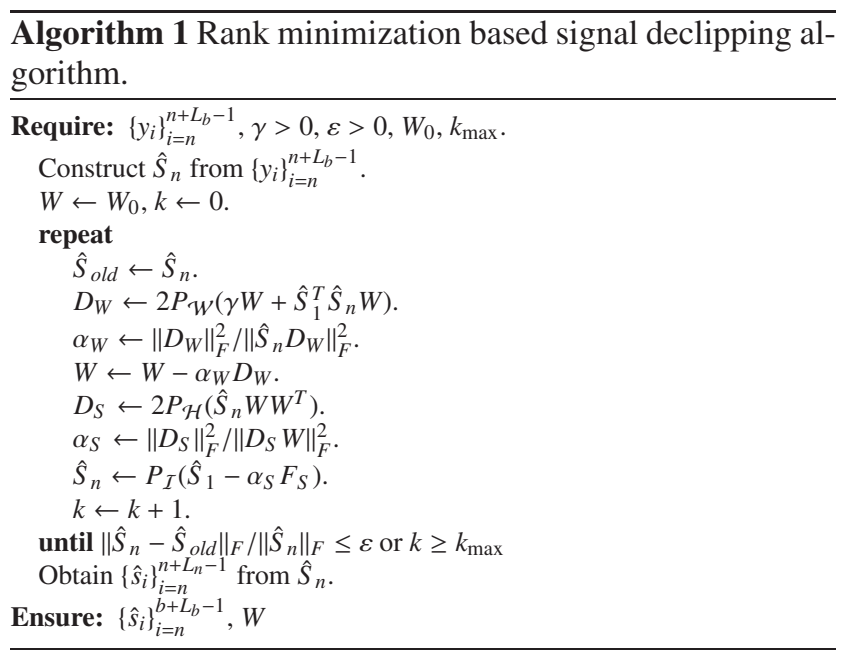




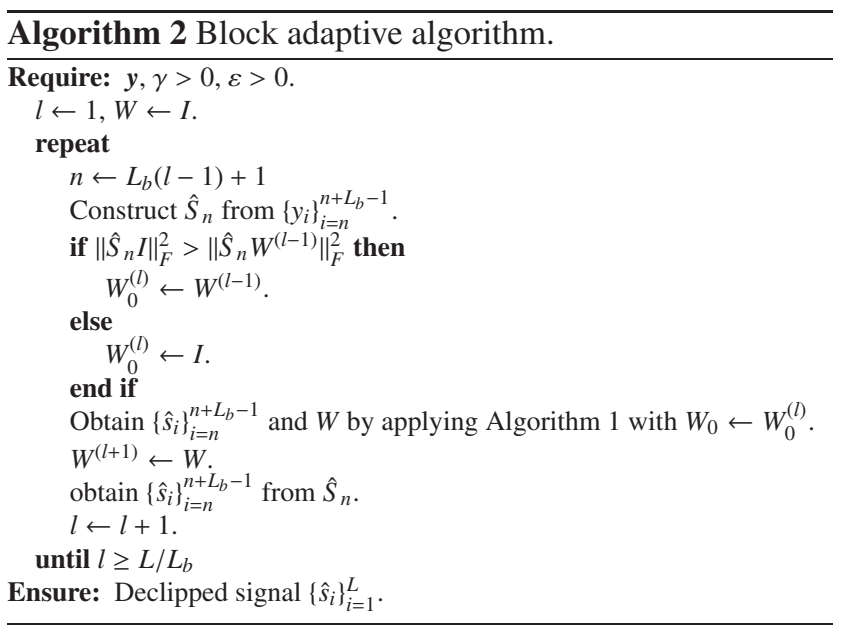

Though this letter deals with non-stationary signals, we assume here that the statistical property of signals mostly changes slightly. This assumption is proper in a lot of applications. For example, in speech and audio signal processing, the signal is quasi stationary in $10-30 \mathrm{msec}$ in general. This quasi stationary assumption implies that the difference between the null space matrices $W$ of neighbor blocks is small. In the other words, we assume that $W$ minimizes the objective function of (8) in the previous block tends to let the value of the present objective function to be small. This motivates us to use $W$ obtained in the previous block as the initial value $W_{0}$ in Algorithm 1. Then this letter proposes the block adaptive algorithm as shown in Algorithm 2, where $\leftarrow$ denotes the substitution operator, and $\left\{y_{i}\right\}_{i=n}^{n+L_{b}-1}$ is a set of observed samples $y_{i}\left(n \leq i \leq n+L_{b}-1\right)$. In this algorithm, to avoid poor quality of the initial value caused by non-stationarity of the signal, $W$ obtained in the previous block is not always used. Because the value of the objective function in (8) with $W=I$ is not greater than that with $W=W^{(l-1)}$ if $\left\|\hat{S}_{n}\right\|_{F} \leq\left\|\hat{S}_{n} W^{(l-1)}\right\|_{F}$, Algorithm 2 uses $W=I$ in such a case.

While it is difficult to give a theoretical proof of improvement by the initial value, numerical examples show that the adaptive algorithm achieves better performance than non-adaptive algorithm. The NSAO algorithm utilizes the null space matrix explicitly, which enables us to implement a block adaptive algorithm in the rank minimization approach.

\section{Numerical Examples}

This section presents numerical examples for the proposed algorithm. We utilize 4 kinds of 6 second speech signals (sampling frequency $=16 \mathrm{kHz}, L=95612$ samples) of University of Tsukuba Multilingual (UT-ML) Speech Corpus, which is available (sample version only) at the web site ${ }^{\dagger}$. These speech signals are generated by 4 women, and their sentences are the same: "A long time ago the north wind

\footnotetext{
${ }^{\dagger}$ http://research.nii.ac.jp/src/eng/list/index.html
}

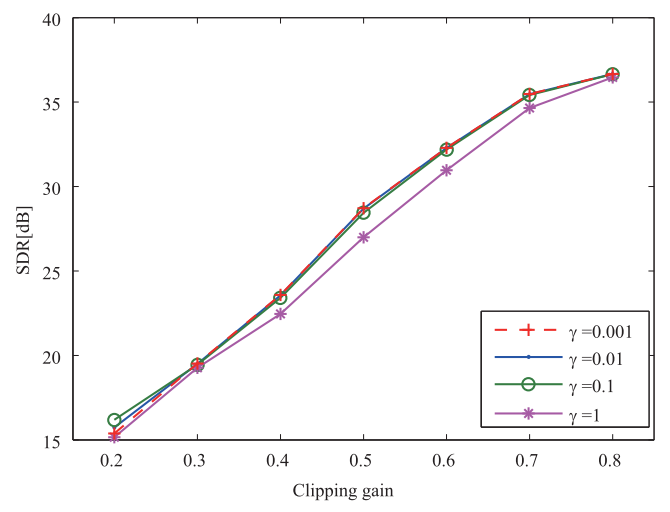

Fig. 1 The performance of the proposed method in several kind of $\gamma$.

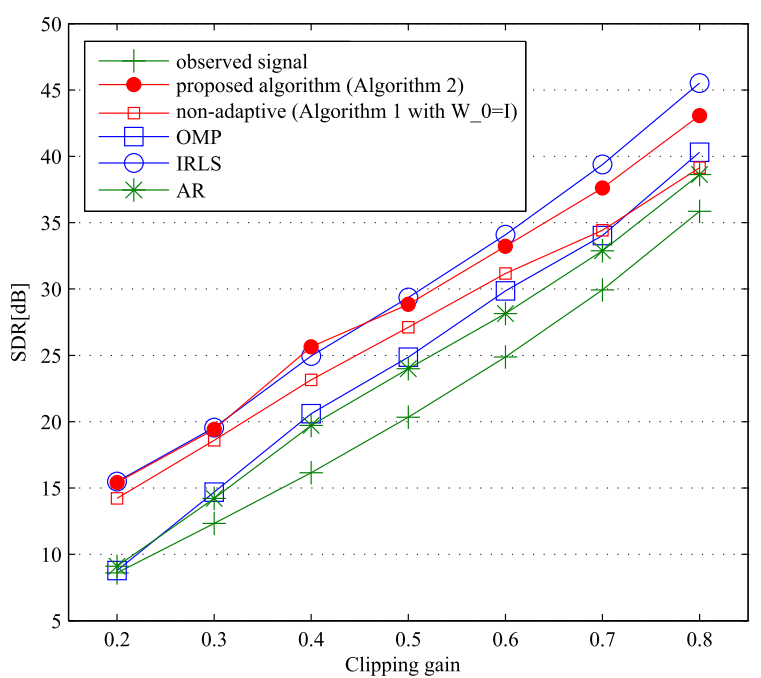

Fig. 2 Average performance of the algorithms for the clipping gain $C=$ $0.2,0.3,0.4,0.5,0.6,0.7$ and 0.8 .

and the sun had a dispute as to who was stronger." We use $N=226, \gamma=0.1, k_{\max }=200$ and $\varepsilon=10^{-2}$ for all experiments.

Figure 1 shows the results of the proposed algorithm with several values of $\gamma$. We can see that the proposed algorithm does not depend on $\gamma$ if $\gamma \leq 0.1$. Thus we use $\gamma=0.1$ in the following experiments. Next, we compare the proposed algorithm with the dual-constrained OMP based algorithm proposed in [4] with given $\theta_{\max }=1$, the iterative reweighted least squares (IRLS) based algorithm, the fixed order AR model based algorithm and Algorithm 2 with non-adaptive block algorithm, where $W_{0}$ is always set as $W_{0} \leftarrow I$. The IRLS based algorithm recovers the clipped signals by solving (6) using the IRLS algorithm proposed in [8] instead of NSAO. In the fixed order AR model based algorithm, the AR coefficients and signals are optimized alternately. These two algorithms are applied with block size $L_{b}=2 N-1=451$, and their terminate condition is that $\left\|\hat{S}_{n}-\hat{S}_{\text {old }}\right\|_{F} /\left\|\hat{S}_{n}\right\|_{F} \leq \varepsilon=10^{-2}$. We examine the cases of the clipping gains $C=0.2,0.3,0.4,0.6,0.7$ and 0.8 . The results are shown in Fig. 2, where the performance of the algorithms are evaluated by the signal-to-distortion ratio 


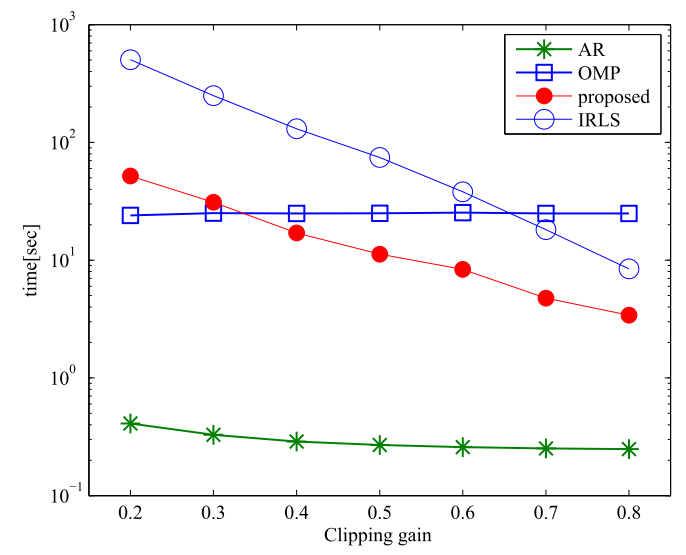

Fig. 3 CPU times of the algorithms per 6 second signal.

(SDR) computed as follows,

$$
\mathrm{SDR}=20 \log _{10}\left(\|\boldsymbol{s}\|_{2} /\|\boldsymbol{s}-\hat{\boldsymbol{s}}\|_{2}\right) .
$$

We can see that SDR of the proposed algorithm is $6 \mathrm{~dB}$ higher than the OMP based algorithm in clipping gain $=0.2$ and achieves signal declipping better than the OMP based and AR model based algorithms in all clipping gains. The proposed algorithm has good performance comparing with the non-adaptive version of Algorithm 2, which implies that the proposed block adaptive algorithm works well. Figure 3 shows the computing time to declipping a 6 second signal. Because the computational cost of the proposed algorithm depends on the number of signals to estimate, the recovery for higher clipping gain requires less computing time. As can be seen, the proposed algorithm much faster than the OMP based algorithm except for $C=\{0.2,0.3\}$ and the IRLS based algorithm. These results indicate that the algorithm can be implemented for real time processing if the clipping gain $C$ is larger than 0.7 .

\section{Conclusion}

This letter deals with the signal declipping problem, which is formulated as the matrix rank minimization problem. To consider the declipping problem of non-stationary signals, this letter proposed the block adaptive signal declipping algorithm based on the NSAO algorithm. Under the assumption that the difference of AR coefficients between neighbor blocks is small, this letter has proposed the block adaptive algorithm where the null space matrix is reused in the NSAO algorithm. Numerical examples show that the proposed algorithm can recover clipped signals efficiently and has better performance than the OMP based algorithm.

\section{References}

[1] J. Tang, "Evaluation of double sided periodic substitution (DSPS) method for recovering missing speech in packet voice communications," Proc. Tenth Annual International Phoenix Conference on Computers and Communications, pp.454-458, 1991.

[2] A. Janssen, R. Veldhuis, and L. Vries, "Adaptive interpolation of discrete-time signals that can be modeled as autoregressive processes," IEEE Trans. Acoust. Speech Signal Process., vol.34, no.2, pp.317-330, 1986.

[3] S.J. Godsill, P.J. Wolfe, and W.N.W. Fong, "Statistical model-based approaches to audio restoration and analysis," J. New Music Research, vol.30, no.4, pp.323-338, 2001.

[4] A. Adler, V. Emiya, M. Jafori, M. Elad, R. Gribonval, and M.D. Plimbley, "A constrained matching pursuit approach to audio declipping," Proc. IEEE ICASSP, pp.329-332, 2011.

[5] T. Takahashi, K. Konishi, and T. Furukawa, "Hankel structured matrix rank minimization approach to signal declipping," Proc. EUSIPCO, pp.1-5, 2013.

[6] T. Ding, M. Sznaier, and O.I. Camps, "A rank minimization approach to video inpainting," Proc. IEEE 11th International Conference on Computer Vision, pp.1-8, 2007.

[7] T. Takahashi, K. Konishi, and T. Furukawa, "Rank minimization approach to image inpainting using null space based alternating optimization," IEEE International Conference on Image Processing, pp.1717-1720, 2012.

[8] K. Mohan and M. Fazel, "Iterative reweighted algorithms for matrix rank minimization," J. Machine Learning Research, vol.13, pp.3441-3473, 2012.

[9] K. Konishi, "Parallel GPU implementation of null space based alternating optimization algorithm for large-scale matrix rank minimization,” Proc. IEEE ICASSP, pp.3689-3692, 2012.

[10] J.F. Cai, E.J. Candés, and Z. Shen, "A singular value thresholding algorithm for matrix completion," SIAM J. Optim., vol.20, no.4, pp.1956-1982, 2010. 